\title{
Consulta de enfermería: un análisis de concepto
}

\author{
Pimentel-Jaimes, José Alfredo ${ }^{1,2 *}$ Casique-Casique, Leticia3; Álvarez-Aguirre, Alicia4; Higuera-Sainz, Jose Luis 1,5;
}

Bautista-Alvarez, Tania Meyatzy ${ }^{6}$

\section{RESUMEN}

Introducción: la consulta de enfermería siempre ha existido en el ámbito público o privado, dentro y fuera de los hospitales y en todos los niveles de atención, sin embargo rara vez se ha implementado de manera sistemática o con este nombre. Objetivo: analizar el concepto de consulta de enfermería. Metodología: el presente análisis se llevó a cabo siguiendo la metodología desarrollada por Walker y Avant, a través de la cual se examinan las características que definen un concepto y sus atributos. Resultados: a partir del presente análisis se propone que, la consulta de enfermería es un servicio técnico, científico, libre y autónomo que se otorga por profesionales de enfermería, ya sea en el ámbito público o privado, dentro y fuera de los hospitales y en todos los niveles de atención sanitaria. Su propósito principal es promover, habilitar, incluir, prevenir, detectar, resolver necesidades y problemas de salud del individuo, familia y comunidad en todas las etapas de la vida. Conclusiones: la importancia de la consulta de enfermería radica en tres aspectos principales: 1) permite resolver necesidades y problemas de salud del individuo, familia y comunidad en todas las etapas de la vida, 2) para los profesionales de enfermería funciona como la puerta de entrada hacia la autonomía profesional, la práctica independiente y el reconocimiento social, y 3) representa un brazo ampliado del sistema nacional de salud (público o privado), ya que aumenta el acceso y la cobertura universal de salud.

\section{Palabras clave: Consulta de enfermería; Enfermería; Sistemas de salud(DeCS, BIREME).}

'Estudiante de Doctorado en Ciencias de Enfermería. Universidad de Guanajuato (Campus Celaya-Salvatierra), México.

${ }^{2}$ Maestro en Ciencias de Enfermería. Universidad Autónoma de Baja California (Campus Mexicali), México. E-mail: alfredo.pimentel@uabc.edu.mx ORCID-ID: https://orcid.org/0000-0003-4384-8370

${ }_{3}^{3}$ Doctora en Enfermería. Universidad de Guanajuato (Campus Celaya-Salvatierra), México. ORCID-ID: https://orcid.org/0000-0002-0532-4819 ${ }^{4}$ Doctora en Enfermería. Universidad de Guanajuato (Campus Celaya-Salvatierra), México. ORCID-ID: https://orcid.org/0000-0001-5538-7364 ${ }_{5}^{5}$ Maestro en Ciencias de Enfermería. Universidad Autónoma de Baja California (Campus Mexicali), México. ORCID:ID: https://orcid.org/0000-0003-18248963

${ }^{6}$ Estudiante de Maestría en Docencia. Instituto Universitario de las Américas y el Caribe, Colima, México. ORCID-ID: https://orcid.org/0000-0002-98673005

Recibido: 05/06/2019

Aceptado: 30/06/2019

*Autor para correspondencia

\section{Cómo citar este artículo}

Pimentel-Jaimes, JA, Casique-Casique L, Álvarez-Aguirre A, Higuera-Sainz JL, Bautista-Alvarez TM. Consulta de enfermería: un análisis de concepto. SANUS. 2019;(10): 70-84. [Acceso_____ ] Disponible en: mes día año 


\title{
Consulta de enfermagem: uma análise do conceito
}

\begin{abstract}
RESUMO
Introdução: Consulta de enfermagem sempre existiu no domínio público ou privado, dentro e fora dos hospitais, e em todos os níveis de atenção. Objetivo: Analisar o conceito de consulta de enfermagem. Metodologia: Esta análise foi realizada seguindo a metodologia desenvolvida por Walker e Avant, através do qual as características que definem um conceito e seus atributos são discuitos. Resultados: Essa análise sugere que a consulta de enfermagem pode ser vista como um serviço técnico, científico, livre e autônomo, concedido por profissionais de enfermagem, quer no domínio público ou privado, dentro e fora dos hospitais e em todos os níveis de atenção. Seu principal objetivo é promover, habilitar, incluir, prevenir, detectar e resolver as necessidades de saúde e os problemas do indivíduo, da família e da comunidade em todas as etapas da vida. Conclusões: A importância da consulta de enfermagem encontra-se em três aspectos principais: 1) permite que as necessidades e problemas de saúde individuais, familiares e comunitários sejam resolvidos em todas as fases da vida; 2) para os profissionais de enfermagem, funciona como porta de acceso à autonomia profissional, à prática independente e ao reconhecimento social, e 3) representa uma extensão do braço do sistema nacional de saúde (público ou privado) à medida que aumenta o acesso à saúde e cobertura universal de saúde.
\end{abstract}

Palavras chave: Consulta de enfermagem; enfermagem; sistemas de saúde (DeCS;BIREME).

\section{INTRODUCCIÓN}

La consulta de enfermería siempre ha existido en el ámbito público o privado, dentro y fuera de los hospitales y en todos los niveles de atención, sin embargo rara vez se ha implementado de manera sistemática o con este nombre. Una de las referencias más antiguas al respecto se remonta hacia el año 1973 en el Johns Hopkins Hospital de Baltimore en Estados Unidos de Norte América, donde las enfermeras especialistas se encargaban de atender y dar seguimiento a personas con problemas crónicos. Estas consultas funcionaban separadamente de las consultas médicas. Aunque había un mecanismo para la remisión a un médico, las enfermeras especialistas controlaban sus consultas de manera libre y autónoma ${ }^{(1)}$. A partir de ahí surge la consulta de enfermería en diferentes países de Europa y el resto de América ${ }^{(2,3)}$.

La consulta de enfermería surge como un paso importante hacia la autonomía profesional y como respuesta a los cambios del panorama socioeconómico, epidemiológico y reformas en las políticas de salud ${ }^{(4,5)}$. A nivel internacional la consulta de enfermería se ha implementado de manera sistematizada (bajo un marco legal vigente) en pacientes con enfermedades agudas y crónicas, adultos mayores y en temas de atención pediátrica y ginecológica(6-9).

A pesar de que ya existe la implementación de la consulta de enfermería, y aunque existen algunas definiciones al respecto(4), hasta la fecha no existe un análisis de concepto de la consulta de enfermería. Dentro de las definiciones existentes en la literatura destacan las siguientes: 1) "la consulta de enfermería es una interacción profesional entre el usuario y la enfermera. La labor de la enfermera se centra en la ayuda al individuo, al grupo familiar y a la comunidad; a realizar autocuidados de salud, del nacimiento a la muerte mediante un proceso de interacción específico y terapéutico"(10); y 2) "la consulta de enfermería, se define como el proceso de interacción que tiene lugar en un espacio físico y temporal determinado entre el usuario y el profesional de enfermería, a través del cual éste proporciona cuidados enfermeros a individuos o grupos con el fin de promover, proteger y restaurar su salud"(11).

La consulta de enfermería además de ser una alternativa importante para el sistema de salud(4); se convierte en una nueva opción para los pacientes en cualquier etapa de la vida, y en cualquier estado dentro del proceso salud-enfermedad, ya que amplía el acceso y la cobertura universal de salud(12). Por otro lado, funciona como la puerta de entrada hacia la autonomía profesional, 
la práctica independiente y el reconocimiento social de nuestra profesión. Por lo anterior, el objetivo del presente estudio es analizar el concepto de consulta de enfermería para diferenciarlo de conceptos como: 1) Gestión de casos; 2) Enfermera de atención primaria; 3) Ejercicio libre de la profesión; 4) Enfermería de práctica avanzada; 5) Enfermeras de enlace; 6) Atención domiciliaria y 7) Consulta médica.

\section{METODOLOGÍA}

El presente análisis se llevó a cabo siguiendo la metodología desarrollada por Walkery Avant ${ }^{(13)}$.Dichos autores argumentan que el análisis de concepto es una vía para la construcción de teoría, que brinda la oportunidad para explicar y describir fenómenos de interés para la práctica de enfermería. Es una estrategia a través de la cual se examinan las características que definen el concepto, es decir, los atributos que lo conforman. Dicha metodología se conforma de las siguientes etapas: 1) selección del concepto; 2) objetivos o propósitos del análisis; 3) identificación de los usos del concepto; 4) la identificación de los atributos; 5) identificación del caso modelo; 6) identificación casos adicionales; 7) identificación de antecedentes y consecuencias; y 8) la definición de los indicadores empíricos, mismas que se desarrollarán en el presente análisis de concepto.

Para la búsqueda y selección de artículos científicos acerca de la consulta de enfermería se revisaron las bases de datos LILIACS, EBSCOhost, PUBMED y el buscador Google Scholar a través de búsqueda básica y avanzada, sin importar el año de publicación. Se utilizaron las siguientes palabras clave en español y su correspondiente en inglés, portugués y francés: análisis de concepto, consulta de enfermería, enfermería, sistemas de salud, además de algunos conceptos relacionados con la consulta de enfermería citados en el apartado de introducción.

\section{RESULTADOS}

\section{Identificación de los usos del concepto}

\section{Definiciones a partir de diccionarios}

Actualmente, en los diccionarios de terminología médica o al menos en la última edición del Diccionario de la Lengua Española(14) no existe el término de consulta de enfermería. Una de las referencias del término encuentra en un diccionario de enfermería en el idioma francés ${ }^{(15)}$, el cual indica que "la consulta de enfermería es un beneficio para informar, asesorar, educar a un paciente o su entorno de salud o de enfermería. La consulta se lleva a cabo en un ámbito hospitalario o no hospitalario, ya sea con receta, a petición del paciente o de enfermeras. Puede ser incluida en una consulta multidisciplinaria".

\section{Revisión de literatura}

Como ya se mencionó en un inicio, la primera referencia respecto a la consulta de enfermería se remonta hacia la década de los 70 en el Johns Hopkins Hospital de Baltimore ${ }^{(1)}$. Posterior a ello, y además de las definiciones ya presentadas ${ }^{(10,11)}$; se proponen otras definiciones:

- Para Boiteux ${ }^{(16)}$ se trata de una "entrega programada de información, educación a un paciente y su familia, respecto a su salud y cuidados de enfermería".

- De acuerdo con Jovic (17) el propósito de la consulta de enfermería es que: "las enfermeras vean los pacientes referidos por un médico (cuidado de heridas, terapia de ostomías, educación, orientación, etc.). Después de la observación, se formalizan las necesidades del paciente, en una perspectiva de la enfermería, la prestación de atención, educar y proporcionar información y asesoramiento dentro de su competencia profesional".

- Galicia, Nájera y Morales(3) refieren que "la consulta de enfermería es un área de oportunidad para la práctica independiente en la que se establece una relación cuidadora y terapéutica, que ayudan a la persona a comprender y actuar sobre su propia salud".

- Según la Sociedad de Enfermería de Atención Primaria de Asturias(18) la consulta de enfermería "consiste en la prestación de cuidados a los usuarios, por demanda directa o programada, en el centro de salud, orientados al mantenimiento y mejora de su salud, a la prevención de la enfermedad, y a proporcionar una respuesta personalizada a las necesidades de salud detectadas".

- Hernández ${ }^{(19)}$ indica que la consulta de enfermería es un "proceso de interacción y de colaboración entre una persona que solicita ayuda para resolver un problema o situación de crisis y un profesional al que aquél supone cualificado para ayudarle". Este autor destaca cuatro características de la consulta de enfermería: 1) la brinda un profesional de enfermería; 2) atiende a personas en lo individual y grupal, con o sin enfermedad; 3) emplea la entrevista directa; y 4) utiliza un sistema de registro.

Por otro lado, dentro de la literatura científica disponible la consulta de enfermería se clasifica en tres tipos: programadas, a demanda y urgentes ${ }^{(11)}$. Se describen a continuación:

\section{1)Consulta programada(9,7)}

a) surge del consenso entre usuario y profesional de enfermería (citas concertadas); b) tiene carácter sistemático y continuado; c) su objetivo es promover, prevenir, detectar y resolver necesidades y problemas de salud de la población fomentando cambios en el estilo de vida y el autocuidado de la salud; d) aplica una metodología científica (Proceso de Atención de Enfermería [PAE]) apoyada en teorías y modelos conceptuales de enfermería para orientar el cuidado de enfermería hacia los usuarios; e) el usuario se convierte en sujeto activo y participativo en su proceso salud-enfermedad; f) favorece la comunicación enfermera-paciente-familia y g) fortalece la comunicación con el equipo multidisciplinario de salud. 


\section{2) Consulta a demanda $a^{(17,20,27)}$}

a) se realiza sin necesidad de cita previa; b) presta atención de enfermería a determinados problemas y/o necesidades de salud mediante la citación directa demandada por el propio usuario u otros profesionales; c) se puede desarrollar en el ámbito público y privado; d) se puede ejercer la investigación, la docencia, el servicio y la práctica independiente; e) se implementan las tecnologías de la información y la comunicación, y tecnologías de aplicación en salud; f) suele utilizarse para resolver problemas de tipo puntual o como entrada para otro tipo de consulta; y g) permite la personalización de los servicios.

\section{3) Consulta urgente (17,22-24). $^{2}$}

a) favorece la rapidez en el acceso y atención de enfermería; b) implica la realización del triage; c) requiere intervención en un plazo de 30 a 120 minutos; d) permite referir al paciente hacia el profesional de salud indicado; y e) demuestra la capacidad de enfermería para resolver autónomamente las demandas de usuarios por patologías agudas y complicaciones crónicas leves.

Aunque se presentan las características por separado de cada uno de los tipos de consulta de enfermería, es importante señalar que estas características no son exclusivas, ya que más de una de las características puede articularse 0 describir perfectamente a los tres tipos de consulta.

\section{Identificación de los atributos}

A partir de las definiciones revisadas y de la literatura científica referente a la consulta de enfermería, se lograron identificar los siguientes atributos del concepto (a continuación se enumeran sin que representen un orden jerárquico):

1) es un proceso de interacción entre el cliente y el profesional de enfermería; 2) proporciona cuidados enfermeros a la persona (sana o enferma), familia y comunidad en todas las etapas de la vida; 3) su objetivo es promover, prevenir, detectar, resolver necesidades y problemas de salud; 4) el usuario se convierte en sujeto activo y participativo en su proceso salud-enfermedad; 5) aplica una metodología científica (PAE) y se apoya en modelos y teorías de enfermería; 6) se implementan las tecnologías de la información y la comunicación, y tecnologías de aplicación en salud; 7) tiene carácter sistemático y continuado; 8) se lleva a cabo en un ámbito hospitalario y no hospitalario, público o privado; 9) se clasifica en tres tipos: programadas, a demanda y urgentes; 10) se utiliza para resolver problemas específicos o como sistema de referencia para otro tipo de consulta; 11) permite la personalización de los servicios de enfermería; 12) demuestra la capacidad de enfermería para resolver de una manera autónoma las demandas de los usuarios por patologías agudas y complicaciones crónicas leves y 13) se ejerce la investigación, la docencia, el servicio y la práctica independiente.

\section{Propuesta de concepto de consulta de enfermería}

La consulta de enfermería es un servicio técnico, científico, libre y autónomo que se otorga por profesionales de enfermería, ya sea en el ámbito público o privado, dentro y fuera de los hospitales y en todos los niveles de atención sanitaria. Su propósito principal es promover, habilitar, incluir, prevenir, detectar, resolver necesidades y problemas de salud del individuo, familia y comunidad en todas las etapas de la vida.

\section{Identificación del caso modelo}

A continuación se presentan tres casos que ilustran el concepto:

\section{Caso modelo}

Meyatzy es una enfermera con más de 20 años de experiencia clínica en el primer y segundo nivel de atención a la salud, ella cuenta con una Maestría con orientación profesionalizante (enfermedades crónicas), tres diplomados, dos certificaciones (una nacional y otra internacional), y un sinnúmero de asistencia a congresos y participación en talleres en el área de profesionalización. En su experiencia ha convivido en innumerables ocasiones con pacientes afectados por la Diabetes Tipo 2 (DT2) y las familias de este, y desea establecer un espacio para efectuar la consulta de enfermería de forma privada en este tipo de pacientes. En la consulta pretende ejecutar sus conocimientos técnicos y científicos de manera libre y autónoma, y que además le genere un ingreso económico adicional a su empleo de base. El propósito de Meyatzy en la consulta de enfermería es promover conductas y estilos saludables en el paciente con DT2 e incluir a su familia en este proceso con el fin de convertirlos en dos elementos activos y participativos, en consecuencia que se habilite a ambos actores en los procedimientos básicos relacionados con el tratamiento de la DT2 (uso de medicamentos, dieta, ejercicio, automonitoreo y salud psicosocial) para el control de la enfermedad, busca además detectar factores de riesgo que contribuyan al desarrollo de complicaciones agudas y prevenir la aparición de complicaciones crónicas en estos pacientes y resolverlos en conjunto o canalizarlo con el profesional de salud si el caso está fuera de su competencia profesional.

\section{Caso límite}

Miguel es un enfermero recién egresado de la especialidad en salud pública, cuanta con 10 años de experiencia clínica y durante el desarrollo de sus prácticas tanto en la Licenciatura como en la Especialidad se dio cuenta que existe una prevalencia muy alta de DT2 en el país y sus consecuencias son fatales. En su experiencia y durante sus estudios se dio cuenta que el sistema de salud se está viendo rebasado por la gran cantidad de pacientes con DT2 y que el tratamiento de 
estos se limita muchas veces a lo farmacológico, es por eso que decidió abrir un consultorio privado de enfermería en el que pretende ejercer libremente su profesión, y orientar a los pacientes sobre su tratamiento completo, es decir, sobre el uso correcto de los medicamentos orales e inyectables, darles varias opciones de alimentos permitidos en la DT2 y sus porciones, como, cuando y durante cuánto tiempo realizar ejercicio, y como automonitorizarse la glucosa por si mismos e interpretar los resultados, y hacer esto mismo con algún familiar si es necesario. El fin último de todo esto, es que a través de la consulta de enfermería Miguel contribuya y haga participe al paciente del control la DT2 y esto permita prevenir las complicaciones de esta.

\section{Caso contrario}

María es una enfermera general que ha dedicado toda su vida profesional (30 años) a la práctica clínica en un hospital de segundo nivel y durante todo ese tiempo trabajó en los servicios de consulta externa, urgencias y medicina interna que comúnmente atiende a pacientes con enfermedades agudas y crónicas. Se sabe que durante su experiencia María ha ejecutado con mucha precisión las indicaciones médicas tanto a pacientes ambulatorios como hospitalizados.

\section{Identificación casos adicionales (conceptos relacionados)}

La consulta de enfermería se relaciona con ciertos conceptos, y aunque no son exactamente lo mismo comparten algunos - la mayoría de los atributos. Entre ellos destacan los siguientes:

- Consulta médica: "es una instancia de encuentro entre el médico y el paciente que representa una de las formas más antiguas de resolver los problemas de saludenfermedad de las sociedades"(25).

- Ejercicio libre de la profesión: "se define como el desempeño de las (os) profesionales de la enfermería, de forma independiente y autónoma, intelectual y financieramente, el cual genera su propia remuneración económica y su estabilidad profesional"(26).

- Enfermería o enfermera de práctica avanzada: "una enfermera titulada que ha adquirido la base de conocimientos de experto, habilidades para la adopción de decisiones complejas y las competencias clínicas necesarias para desarrollar un ejercicio profesional ampliado cuyas características vienen dadas por el contexto o el país en el que la enfermera está acreditada para ejercer. Como nivel de acceso se recomienda un título universitario de posgrado de nivel máster"(27).

- Enfermera de atención primaria o comunitaria: "aplica de forma integral un conjunto de cuidados al individuo, la familia y la comunidad, es decir, debe contribuir a que las personas adquieran habilidades, hábitos y conductas que fomenten su autocuidado en el marco de una atención primaria integral y comunitaria que incluye la promoción, protección, recuperación y rehabilitación de la salud, y prevención de la enfermedad"(28).

- Enfermera gestora de casos o de enlace: "consiste en la atención y provisión de cuidados a la persona y cuidador/a, planificación, coordinación y activación de recursos, apoyo, facilitación y promoción, participación, formación, información, educación para la salud, investigación y evaluación de resultados en salud"(29).

- Continuidad asistencial o de cuidados: "se trata de satisfacer las necesidades socio-sanitarias y las expectativas de una población mejor informada y más autónoma"(30). "Implica contemplar el tratamiento del paciente como un todo, en un sistema de atención integrado con el propósito de que el plan de cuidados de un paciente progrese sin interrupciones, los servicios deben ser continuos y estar coordinados"(31).

\section{Identificación de antecedentes y consecuencias de la consulta de enfermería}

Hoy en día existe un importante cambio en el panorama epidemiológico mundial dado por la disminución y manejo eficiente de las enfermedades agudas y el incremento de las enfermedades crónicas como consecuencia de una infinidad de factores, entre ellos los conductuales, los relacionados con el estilo de vida y el envejecimiento poblacional|(32,33). Dicho panorama no solo vislumbra el problema actual, sino que además exige un cambio en el acceso y la cobertura universal de salud de los países para brindar atención de alta calidad ${ }^{(34)}$. En este sentido la consulta de enfermería surge como una alternativa importante, ya que por un lado se esperaría que contribuya con el acceso y la cobertura universal de salud en todos los niveles de atención y por otro, permitiría el trabajo independiente y la autonomía de los profesionales de enfermería.

Existen importantes indicios de que la consulta de enfermería tiene resultados positivos cuando se ejecutan intervenciones en poblaciones vulnerables, en pacientes con enfermedades agudas y crónicas, y en temas de atención pediátrica y ginecológica ${ }^{(6-9)}$. Y aunque todavía se está trabajando en la sistematización; dentro de las herramientas metodológicas que destacan es el PAE, esta herramienta permite valorar, diagnosticar, planificar, ejecutar y evaluar las intervenciones de enfermería en la práctica asistencial de una forma estructurada, homogénea, lógica y sistemática ${ }^{(35)}$. Además de lo anterior, es importante señalar que la consulta de enfermería en su forma cercana al ejercicio libre de la profesión permite a los profesionales de la enfermería, actuar de forma independiente y autónoma, intelectual y financieramente, el cual genera su propia remuneración económica y su estabilidad profesional(21).

\section{Definición de los indicadores empíricos}

Más que realizar una medición del concepto a través de algún indicador empírico; se han empleado algunas estrategias y formas de organizar la consulta de enfermería ${ }^{(36,37)}$ 
instrumentos auto elaborados según la necesidad que se trate en la consulta ${ }^{(6,8,38,39)}$ y algunos de ellos basados en algún referente teórico de enfermería, pero el instrumento por excelencia para la implementación y sistematización de la consulta de enfermería es el Proceso de Atención de Enfermería(40-42), ya que ha demostrado la capacidad de orientar de manera eficaz los cuidados enfermeros.

\section{CONCLUSIONES}

La importancia de la consulta de enfermería radica en tres aspectos principales: 1) permite resolver necesidades y problemas de salud del individuo, familia y comunidad en todas las etapas de la vida, 2) para los profesionales de enfermería funciona como la puerta de entrada hacia la autonomía profesional, la práctica independiente y el reconocimiento social, y 3) representa un brazo ampliado del sistema nacional de salud (público o privado), ya que aumenta el acceso y la cobertura universal de salud, y asegura la calidad de atención a través de la sistematización de la misma. Por lo tanto, se espera que en los próximos años los profesionales de enfermería, no solo se encuentran familiarizados con la consulta de enfermería, sino que además establecen la consulta de enfermería general y especializada con los registros legales y sanitarios correspondientes, y que además de contribuir con las estrategias de salud de su país; permita resaltar el quehacer de enfermería ante la sociedad.

\section{CONFLICTO DE INTERESES}

Los autores declaramos no tener ningún conflicto de intereses.

\section{FINANCIAMIENTO}

El presente estudio no contó con una fuente de financiamiento.

\section{REFERENCIAS BIBLIOGRÁFICAS}

1. Allison SE. A framework for nursing action in a nurseconducted diabetic management clinic. Journal of Nursing Administration [Internet]. 1973; 3(4): 53-60. Disponible: doi: 10.1097/00005110-197307000-00016

2. Guirao JA. La consulta de enfermería: aparición, implantación y desarrollo en España. [Internet]. 1998 [consultado 01 de Febrero de 2019]. Disponible en: http:// www.uv.es/joguigo/materiales-dominio-profesional/cde. html

3. Galicia-Aguilar RM, Nájera-Gutiérrez G, Morales-Nieto A. Consulta de enfermería en la atención primaria. Revista de Enfermería del Instituto Mexicano del Seguro Social. 2010; 18(1): 31-34.
4. Warchol N. La consultation infirmière: un pas vers l'autonomie professionnelle. Recherche en soins infirmiers. 2007; (4): 76-96.

5. García L, Martín M. Consulta de enfermería programada en un centro de medicina familiar. Enfermería: Cuidados Humanizados. 2016; 1(1): 43-48.

6. Catafesta G, Poletto DK, Franco ES, Pedroso BC, Delacanal DS. Consulta de enfermería ginecológica en la estrategia salud de la familia. Archivos de Ciencias de la Salud. 2015; 22(1): 85-90.

7. Martínez-González NA, Djalali S, Tandjung R, HuberGeismann F, Markun S, Wensing M, Rosemann T. Substitution of physicians by nurses in primary care: a systematic review and meta-analysis. BMC health services research. 2014; 14(1): $1-17$.

8. Moraes JT, da Fonseca DF, da Mata LRF, de Oliveira PP, de Castro SF, da Silva JF. Validação de um instrumento para consulta de enfermagem à pessoa com diabetes mellitus e/ ou hipertensão arterial. Revista de Enfermagem Referência. 2018; 19(4): 127-136.

9. Souto MC, Zaccara AAL Fernandes MA, Santos de Platel IC, Albuquerque TM, Felix ZC. Consulta de enfermería para el niño y el anciano: revisión integrativa de la literatura. Diario de Ciencias de la Salud. 2015; 18(3): 241-248.

10. Jiménez Otero MO, Ruiz Arias E. Consulta de Enfermería en consultorios y ambulatorios. Sevilla: Dirección General de Atención Primaria y Promoción de la Salud. Consejería de Salud, 1986.

11. Sánchez MJ, Cayuela FPS, Delgado GMS, Lifante PZM, Morales MI. Introducción a las competencias de la Enfermería Familiar y Comunitaria y su sistema de registro en OMI-AP [Internet]. 2015 [consultado 02 de Febrero de 2019]. Disponible en: https://www.murciasalud.es/recursos/ ficheros/342237-libro_seapremur.pdf

12. Cassiani SHDB, Rosales LK. Initiatives towards Advanced Practice Nursing Implementation in the Region of the Americas. Escola Anna Nery. 2016; 20(4): 1-2.

13. Walker LO, Avant KC. Strategies for theory construction in nursing. Boston: Prentice Hall 5th edition, 2011.

14. Real Academia Española. Diccionario de la lengua española [Internet]. RAE. 2014 [consultado 10 de Enero de 2019]. Disponible en: http://dle.rae.es/

15. Magnon R, Lepesqueux M, Dechanoz G. Dictionnaire des Soins Infirmiers. Paris: Editions Amiec recherché, 1995. 
16. Boiteux A. La consultation infirmière, une avancée pour la profession. Soins. 2006; 707: 31.

17. Jovic L. La consultation infirmière, la gestion des compétences dans un système complexe. Ljilijana JOVIC [Internet]. 2000 [consultado 15 de Enero de 2019]. Disponible en: http://fulltext.bdsp.ehesp.fr/ Ensp/Memoires/2000/ig/ jovic.pdf

18. Sociedad de Enfermería de Atención Primaria de Asturias. Análisis de la situación y propuestas de mejora de la enfermería de atención primaria en Asturias [Internet]. SEAPA. 2004 [consultado 26 de Enero de 2019]. Disponible en: http:// www.seapaonline.org/uploads/documentacion/Informes/ Analisis\%20de\%20la\%20situacion\%20y\%20propuestas\%20 de\%20reforma\%20de\%20la\%20enfermeria\%20de\%20 A.P.\%20en\%20asturias.pdf

19. Hernández JC. Salud Pública y Atención Primaria de Salud: la consulta de enfermería [Internet]. Universidad de Cantabria. 2017 [consultado 16 de Enero de 2019]. Disponible en: http://ocw.unican.es/ciencias-de-la-salud/salud-publicay-atencion-primaria-de-salud/ material-de-clase/bloqueiv/4.5_consulta_enfermeria.pdf

20. Rodríguez MG, Serrano MM, Ibáñez GA, Perdikidi GA, Ariza CG, Martín FJ. Análisis de la consulta enfermera de Atención Primaria en la Comunidad de Madrid (España). Enfermería Clínica. 2018; 28(6): 347-404.

21. Guillermina AM, Mori, FMLV. Empoderamiento de las mujeres a través del ejercicio libre de la enfermería [Internet]. IPN. 2015 [consultado 01 de Febrero de 2019]. Disponible en: http://www. genero.ipn.mx/Difusion/Documents/mtc7.pdf

22. Brugués $A B$, Grao $A P$, Rodríguez FP, Viladomat EM, Ferret JG, Mateo GF. Evaluación de la gestión enfermera de la demanda en atención primaria. Atención Primaria. 2016; 48(3): 159-165.

23. Sánchez RC. Enfermería en la clasificación de pacientes en urgencias: dificultades y propuestas de mejora. Revista Enfermería CyL. 2018; 10(2): 17-27.

24. Evangelista F, García BE, Vega GR. Implantación de una consulta de enfermería para la gestión de la demanda urgente en un centro de Atención Primaria. Revista Iberoamericana de Educación e Investigación en Enfermería 2015; 5(2):48-57.

25. Castro R. Teoría social y salud [Internet]. 2011 [consultado 18 de Enero de 2019]. Disponible en: http://www.crim.unam. mx/ web/sites/default/files/Teor\%C3\%ADa\%20social\%20 y\%20 salud_0.pdf

26. Aguilar AG, Cataño FV, Tlalpan BA, Nieto AM, Solano
BG. Proceso de aprehensión de identidad profesional en enfermería. Revista Cuidarte. 2018; 9(3): 1-2.

27. Bryant $L D$, et al. Framework for evaluating the impact of advanced practice nursing roles. Journal of Nursing Scholarship. 2016; 48(2): 201-209.

28. Dandicourt TC. Competencias profesionales para el especialista de Enfermería Comunitaria en Cuba. Revista Cubana de Enfermería. 2016; 32(1): 1-12.

29. Phillips C. Enfermería y el modelo asistencial "gestión de casos". Revista Uruguaya de Enfermería. 2015; 10(2): 1-8.

30. Casado MC. El informe de continuidad de cuidados como herramienta de comunicación entre atención hospitalaria y atención primaria. Revista Española de Comunicación en Salud. 2014; 5(2): 102-111.

31. Calvo MJ, Rodríguez JI, Villarubia CS, López ER, Maldonado JM. La enfermera de atención primaria como garante de la continuidad de cuidados: Intervención proactiva tras el alta hospitalaria. RISAI-Revista de Innovación Sanitaria y Atención Integrada. 2015; 7(1): 1-7.

32. Organización Mundial de la Salud (OMS). Salud en las Américas. Resumen: panorama regional y perfiles de país [Internet]. OMS. 2017 [actualizado 2017; consultado 15 de Marzo de 2019]. Disponible en: http://iris.paho.org/xmlui/ handle/123456789/34322

33. Organización Mundial de la Salud (OMS). Estadísticas sanitarias mundiales [Internet]. OMS. 2014 [actualizado 2014; consultado 23 de Enero de 2017]. Disponible en: http://apps. who.int/iris/bitstream/10665/112817/1/WHO_ HIS_HSI_14.1_ spa.pdf

34. Zug KE, Cassiani SHDB, Pulcini J, Garcia AB, Aguirre-Boza $F$, Park J. Advanced practice nursing in Latin America and the Caribbean: regulation, education and practice. Revista Latino-Americana de Enfermagem. 2016; 24: 1-9.

35. Actualización enfermera. Metodología Enfermera [Internet]. Actualización enfermera. 2017 [consultado 24 de Enero de 2019]. Disponible en: http://enfermeriaactual.com/ metodologia-enfermera/

$\mathrm{s}$

36. Fernández MP, García GL, García EG, Gérvas JJ. Contenido y organización de una consulta de enfermería. Aten Primaria. 1988; 5: 272-276.

37. Forero VJ, Barrios AS. Rol de enfermería en la consulta de prediálisis en el paciente con enfermedad renal cónica avanzada. Enfermería Nefrológica. 2016; 19(1): 77-86.

38. Manzini FC, Simonetti JP. Consulta de enfermagem aplicada a clientes portadores de hipertensão arterial: uso da 
teoria do autocuidado de Orem. Revista Latino-Americana de Enfermagem. 2009; 17(1), 113-119.

39. García L, Martín M. Consulta de enfermería programada en un centro de medicina familiar. Enfermería: Cuidados Humanizados. 2016; 1(1), 43-48.

40. Jordán AAl, Estrada RD. Rol enfermero en la valoración de síndromes geriátricos y estado funcional en una consulta de geriatría. Gerokomos. 2018; 29(4): 160-164.

41. Martín RA. Proceso de atención de enfermería ante un paciente diagnosticado de diabetes mellitus tipo 2. Archivos de Medicina Familiar y General. 2018; 11(2): 15-20.

42. Reyes-Caballero MC, Obregón-Pérez, N, Gálvez-Morfa, R, Pérez-Pérez, A. Capacitación para el desarrollo de la consulta de enfermería en la atención primaria de salud. Edumecentro. 2018; 10(3), 106-121. 